\title{
ANALISIS
}

\section{IMPACTO DE LAS REDES SOCIALES EN EL PERIODISMO}

\section{The impact of social media on journalism}

\author{
Francisco Campos-Freire, José Rúas-Araújo, Xosé López-García y Valentín- \\ Alejandro Martínez-Fernández
}

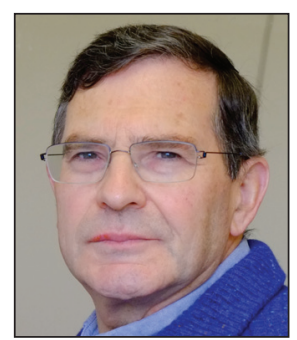

Francisco Campos-Freire es doctor por la Universidad de Santiago de Compostela (USC) y profesor de su Facultad de Ciencias de la Comunicación desde 1991. Es miembro del Grupo de Novos Medios de la USC, coordinador de la Red Internacional de Investigación de Gestión de los Medios de Comunicación (R2014/026 Xescom) e investigador del Programa Prometeo (2014-2016) del Senescyt en las Universidades Técnica Particular de Loja y Pontificia Católica de Ibarra en Ecuador. http://orcid.org/0000-0003-1831-1838

Universidad de Santiago de Compostela, Facultad de Ciencias de la Comunicación Avda. Castelao, s/n. 15705 Santiago de Compostela, España francisco.campos@usc.es

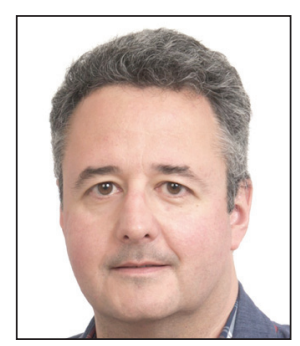

José Rúas-Araújo es profesor titular de la Facultad de Ciencias Sociales y de la Comunicación de la Universidad de Vigo y coordinador del grupo de investigación Necom: neurocomunicación, publicidad y política. Sus ámbitos de investigación son la comunicación política, electoral e institucional y las neurociencias aplicadas a las ciencias sociales y la persuasión.

http://orcid.org/0000-0002-1922-9644

Universidad de Vigo, Facultad de Ciencias Sociales y de la Comunicación Campus A Xunqueira, s/n. 36005 Pontevedra, España http://necom.uvigo.es joseruas@uvigo.es

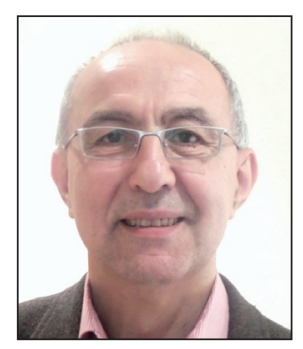

Xosé López-García es catedrático de periodismo en el Departamento de Ciencias de la Comunicación de la Universidad de Santiago de Compostela, doctor en historia y periodista. Coordina el grupo de investigación Novos Medios. Entre sus líneas de investigación están el estudio de los medios digitales e impresos, el análisis del impacto de la tecnología en la comunicación mediada, el análisis del funcionamiento de las industrias culturales, y la estrategia combinada de productos impresos y online en la sociedad del conocimiento.

http://orcid.org/0000-0002-1873-8260

Universidad de Santiago de Compostela, Facultad de Ciencias de la Comunicación Avda. Castelao, s/n. 15782, Santiago de Compostela, España xose.lopez.garcia@usc.es

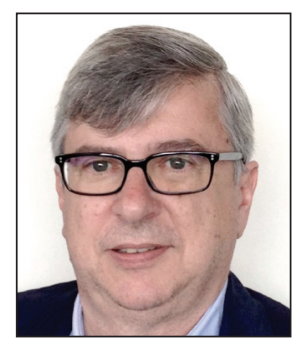

Valentín-Alejandro Martínez-Fernández es licenciado en ciencias de la información por la Universidad Complutense de Madrid (UCM) y Master MBA en dirección y administración de empresas por la Universidad de A Coruña (UDC). Doctor en ciencias de la información por la UCM, es profesor titular de universidad en la Facultad de Ciencias de la Comunicación y en la Facultad de Economía y Empresa de la UDC. Ha sido director del diario El ideal gallego y directivo en otras empresas informativas. Es autor de artículos sobre medios de comunicación y estrategias de comunicación. Investigador del Programa Prometeo (2015-2016) de la Secretaría de Educación Superior, Ciencia, Tecnología e Innovación de la República del Ecuador, en la Universidad Técnica Particular de Loja. http://orcid.org/0000-0003-0069-675X

Universidad de A Coruña, Facultad de Ciencias de la Comunicación Campus de Elviña, s/n. 15071 A Coruña, España valejand@udc.es

\section{Resumen}

Metamedios y redes de valor son dos conceptos emergentes en el actual sistema de comunicación. El primero se refiere a la transformación informática de los procesos y contextos mediáticos, tanto de producción como de uso de los medios; 
y el segundo al sistema que valoriza los flujos de información, conocimiento y relaciones sociales de la comunicación. La revolución de los metamedios y las redes digitales es bien patente en los nuevos usos de las personas, las organizaciones y las estructuras de los medios tradicionales de comunicación. Y éstos ponen las redes -Twitter, Facebook y YouTube principalmente- en el objetivo de la aportación de valor, como se ve en los datos y análisis que se presentan en este artículo. Se ofrecen los resultados del Barómetro de gestión de medios, una encuesta realizada a finales de 2015 a 300 empresas de comunicación de España sobre su transformación metamedia y el uso de las redes sociales digitales.

\title{
Palabras clave
}

Metacomunicación; Metamedios; Medios tradicionales; Redes sociales digitales; Redes de valor; Barómetro de gestión de los medios de comunicación.

\begin{abstract}
Metamedia and value networks are two emerging concepts in the communication area. The first one refers to the computing transformation of media processes and contexts, both in production and use of the media; the second one relates to the system encouraging information flows, knowledge, and communication social relationships. The revolution of metamedia and digital networks is to be seen in the way people use and consume information, as well as organizations and traditional media outlets. They understand the networks - mainly Twitter, Facebook, and YouTube- as the main value providers, as the present study concludes. This work offers the results of the Media management barometer, a survey undertaken at the end of 2015 and administered to 300 Spanish media companies to check their metamedia transformation and the use of digital social networks.
\end{abstract}

\section{Keywords}

Meta-communication; Metamedia; Traditional media; Digital social networks; Value networks; Media management barometer.

Campos-Freire, Francisco; Rúas-Araújo, José; López-García, Xosé; Martínez-Fernández, Valentín-Alejandro (2016). "Impacto de las redes sociales en el periodismo". El profesional de la información, v. 25, n. 3, pp. 449-457.

\section{Introducción}

Los metamedios impulsan la revolución digital. Así lo indican los resultados de la encuesta del Barómetro de medios de 2015, realizado por la Red Internacional de Investigación de la Gestión de la Comunicación (Xescom), constituida por cuatro grupos de investigación de las universidades públicas de Galicia, y que agrega también a otros diez equipos de España, Portugal, Brasil, Ecuador y México.

El objeto de estas investigaciones es desentrañar conceptos y estudiar los cambios que se están produciendo tanto en la gestión de los procesos, contextos y estructuras de la comunicación como en el uso de los productos de las mismas. Escogimos los metamedios y las redes digitales porque son, sin duda, aspectos centrales de los procesos de transformación que están experimentando los medios tradicionales de comunicación, en particular, y las organizaciones sociales, en general. Por eso planteamos en el $\mathrm{Ba}$ rómetro de gestión medios de comunicación las siguientes preguntas:

- ¿Cree que las redes sociales digitales han cambiado los medios de comunicación tradicionales?

- ¿Cuál de las redes sociales digitales cree que le aporta o puede aportar en el futuro más valor a su medio y/organización de comunicación?

- ¿Cree que las redes sociales están cambiando o cambiarán en el futuro inmediato el periodismo?

\section{Metodología}

La metodología de elaboración del Barómetro de medios se basa en una encuesta de una muestra aleatoria de 300 empresas representativas de la diversidad del sector (prensa, radio, televisión y medios digitales), y en el análisis de los resultados de doce cuestiones por parte de un grupo de expertos en cibermedios de la Red Xescom. El perfil empresarial de la muestra es de un $49,1 \%$ de organizaciones de menos de 10 empleados, un $37,7 \%$ de 10 a 49, un $9,4 \%$ de 50 a 250 , y un $3,8 \%$ de más de 250 .

La fiabilidad y validez de los instrumentos y las medidas quedan establecidas en base al procedimiento estadístico para este tipo de investigaciones. De acuerdo con los resultados (de las 300 respondieron 106), el margen máximo de error sobre el total de la muestra es del 9\% (2sigma), lo que ha permitido trabajar con datos fiables en la segunda parte, en el análisis de resultados y su posterior interpretación.

La hipótesis de la que se ha partido consiste en el reconocimiento por parte de los responsables de los medios de comunicación de la importancia creciente del papel de los metamedios a la hora de alimentar redes de valor en el actual modelo de la sociedad en red. El objetivo prioritario del apartado del Barómetro recogido en este artículo tenía como finalidad medir la opinión sobre el cambio que han impulsado las redes en los medios tradicionales. Conocer el punto de vista sobre el uso de las redes para conservar y 
proyectar el valor de los medios tradicionales. Indagar sobre las que impactan en los negocios de los medios de comunicación y saber más sobre la relación que los responsables de estas empresas establecen entre ellas y el futuro del periodismo.

Con tal finalidad se han definido las preguntas, tras la experiencia de encuestas anteriores, y se ha perfilado el instrumento de medición que, de forma periódica, aplica el Barómetro de gestión de medios de comunicación, que es una actividad que realiza cada dos años el Grupo de Novos Medios de la Universidad de Santiago y la Red Internacional de Investigación de Gestión de la Comunicación (Xescom), coordinada desde el citado grupo compostelano. El primer Barómetro se llevó a cabo en 2012 y el segundo fue realizado entre los meses de agosto y noviembre de 2015. Esta última encuesta constaba de doce preguntas y fue realizada telefónicamente, con refuerzo de envío de la misma por correo electrónico, a los máximos gestores (directores o directores generales) de las empresas de comunicación o responsables en quienes ellos delegaron la función de responder.

Un metamedio es un medio online generado por ordenador, con propiedades de los medios tradicionales y otras nuevas potencialidades basadas en la computación, que tiene autonomía para la creación, edición, reproducción, difusión, acceso y descarga (navegación, búsqueda, lectura, escucha, visualización e interacción) de distintos contenidos multimedia

\section{Algunos conceptos básicos}

El concepto de metamedios es más preciso que el de medios digitales o nuevos medios, más antiguo e incluso más amplio que el de cibermedio. Su genealogía se remonta al concepto de simulación de la máquina de Alan Turing en los años 30 del siglo pasado, al anticipo de la teoría mediática de McLuhan (1964) y a su materialización a través de las investigaciones sobre informática y ordenadores desarrolladas por Sutherland, Engelhard, Kay y otros. Según Manovich (2015), el paradigma metamedia está en el centro de la cultura del ordenador y su lógica trasciende a los procesos, contextos y tendencias postmodernas (remezclas, mestizajes e hibridaciones) de la globalización (García-Canclini, 1989).

Un metamedio es un medio digital online generado por ordenador, con propiedades de los medios tradicionales y otras nuevas potencialidades basadas en la computación, que tiene autonomía para la creación, edición, reproducción, difusión, acceso y descarga (navegación, búsqueda, lectura, escucha, visualización e interacción) de distintos contenidos multimedia (Jensen, 2013; Campos-Freire, 2015a; 2015b). Pero el concepto traspasa de la noción técnica de digitalización, como ocurre en la convergencia estructural y cultural (Gordon, 2003; Jenkins, 2006), al ambiente donde se juntan metacomunicación, metalenguajes y metadatos.
Los metamedios utilizan la materia prima de los medios tradicionales y la evolución de las técnicas informáticas "existentes o aún no inventadas" para reinventarse continuamente, como señala Manovich $(2005,2015)$, quien también destaca que "nunca la innovación fue tan simple y sencilla" (una persona con un ordenador se convierte en metamedio). Este mismo autor (Manovich, 2008), que toma el concepto de Kay y Goldberg (1977), describe cuatro etapas de evolución metamedia: experimentación e innovación (1960-1970); aplicación masiva y comercialización (19801990); convergencia e hibridación (1990-2003); y redes de medios sociales (a partir de 2004).

Los metamedios producen distintas variedades de meta comunicación (de uno a uno, de uno a muchos, de muchos a muchos) y potencialidades para la interacción social masiva (redes digitales) o la inmersión en los entornos virtuales mediante la cohesión, coherencia, compromiso y aumento de la densidad social (Guitton, 2012). Algunas de esas características ya fueron señaladas por Bateson (1973) al estudiar a los seres humanos como metamedios de primer grado, de quienes los medios y las redes digitales toman las potencialidades de interacción (Jensen, 2010). Pero el ambiente de la metacomunicación hay que extenderlo no sólo a los actores humanos sino también a los artefactos técnicos o actantes (Latour, 1987, 1993) de gestión de metadatos (robots, motores de búsqueda e internet de las cosas), realidad aumentada y virtual.

Los medios tradicionales, los metamedios y las redes digitales conforman la arquitectura de la sociedad de la comunicación a través de la que se relacionan millones de ciudadanos, organizaciones y otros actores sociales. Esta arquitectura se equipara a un ecosistema por su complejidad, interdependencia, diversidad de especies y autoorganización. La arquitectura del ecosistema no sólo relaciona a los medios con sus audiencias sino también a éstas entre sí y con otras organizaciones que se han convertido en metamedios para comunicarse con sus públicos, clientes, usuarios y partes interesadas. El producto de esas interacciones es capital relacional y social.

Los metamedios utilizan la materia prima de los medios tradicionales y la evolución de las técnicas informáticas "existentes o aún no inventadas" para reinventarse continuamente

Ese nuevo ambiente tecnocultural no sólo trastoca las formas de relacionarse y comunicarse sino también los modelos de organización, producción, comercialización y apropiación de las distintas formas de valor de casi todas las industrias, pero singularmente de las relacionadas con la información, la comunicación y la cultura. Por eso los medios tradicionales miran con preocupación y atención a los metamedios y a las redes, para tratar de recuperar parte del valor que se les escapa por esas cañerías.

Los sitios de redes digitales (social network sites), que surgen de la web 2.0, se han convertido en plataformas de co- 
municación y relación social en torno a las que se articulan ecosistemas mediáticos (Boyd; Ellison, 2007; Beer, 2008; Stenger, 2009; Campos-Freire, 2015a; 2015b). Las redes son ecosistemas metamedia de perfiles digitales individuales o de organizaciones, públicos o semipúblicos, que permiten establecer relaciones e intercambios de flujos de contenidos propios o ajenos, conversaciones, prescripciones, transacciones, experiencias y uso de dispositivos de inteligencia artificial con fines particulares, sociales, comerciales o institucionales. Primero se desarrollaron las redes generalistas (MySpace, Facebook, Twitter) y luego las temáticas (de negocios, contactos, científicas, etc.).

\section{Los medios tradicionales miran con} preocupación y atención a los metamedios y a las redes, para tratar de recuperar parte del valor que se les escapa por ellas

La herramienta es el metamedio, la plataforma la red y el ecosistema su arquitectura. La red es de valor cuando existe un grupo intencionado de personas $u$ organizaciones creadoras de bienes económicos y sociales mediante intercambios dinámicos complejos tangibles e intangibles (Allee, 2009). El objetivo de la red de valor es generar beneficio económico o de otro tipo para sus participantes. La teoría y el análisis de la red de valor es una forma orgánica y precisa para describir, analizar y evaluar el capital social (Coleman, 1998; Lin, 2001; Ellison; Vitak; Gray; Lampe, 2014) e integrar el desempeño de la organización en ambientes complejos.

\section{Estudiar la complejidad de las redes}

En un ecosistema comunicativo cada vez más complejo, la industria de la comunicación no tiene dudas: el presente y el futuro está en la sociedad enredada. Cuando los nuevos medios digitales entran en su tercera década de evolución, ahora revestidos de metamedios, nadie quiere vivir al margen de las redes sociales. A lo largo de los últimos años ha habido importantes cambios con los multimedia, la web social, la comunicación móvil, la gestión de los big data, las nuevas formas de uso y consumo, la innovación en los modelos de negocio y sistemas de audimetría, la experimentación de la realidad virtual y la conexión a la internet de los objetos. Al igual que en la empresa privada, la innovación también se persigue como uno de los objetivos de salvación del servicio público audiovisual europeo (Donders; Raats, 2012; Suárez-Candel, 2012; Tambini, 2015). En este aspecto hay muchos retos de investigación tanto para la industria como para la academia.

Desde el mismo momento de popularización de las redes sociales, los científicos sociales multiplicaron sus aproximaciones para intentar desentrañar los nuevos procesos comunicativos en red. Las investigaciones, tras la definición del concepto (Boyd; Ellison, 2007) y la metodología para su estudio (Carrington; Scott; Wasserman, 2005), analizaron la naturaleza y tipo de relación en las redes (Hargittai, 2007), las motivaciones de los usuarios (Gangadharbatla, 2008), la influencia mutua entre las personas que están en las redes sociales (Christakis; Fowler, 2009) o cómo se han converti- do en fuente informativa para muchas personas (Thelwall, 2008, Lee; Ma, 2012), entre otros aspectos. A día de hoy las investigaciones y publicaciones se cuentan por millares.

En el año 2009 se publicaron varios trabajos sobre la explosión de Twitter y Facebook como redes de comunicación (Farhi, 2009; Greenhow; Reifman, 2009; Lowery, 2009). Desde el año 2010 se conocen los resultados de varias investigaciones que exploraban el impacto de los medios sociales en el periodismo (Harper, 2010) y el uso mayoritario que hacen periodistas de la Europa Atlántica -Portugal, España y Francia- (Herrero, 2013; Veloso, 2015) y de los principales países (Oriella PR Network, 2011; Willnat; Heaver, 2014; Cision, 2015).

En los últimos cinco años, la mayoría de las redacciones de los medios buscaron con diferentes estrategias su presencia en las redes sociales (Lasorsa; Lewis; Holton, 2011; Paulussen; Harder, 2014), lo que ha supuesto un nuevo panorama para los medios, generando incipientes posibilidades y ventajas, pero también nuevas exigencias y requerimientos (García-Estévez, 2013). A fin de dar respuesta a los nuevos desafíos, se multiplicaron las investigaciones sobre las redes más empleadas por los periodistas y los medios, así como acerca de su uso, (Kim; Kim; Nam, 2014), en especial de Twitter y Facebook, presentes en casi todas las iniciativas mediáticas.

\section{Ningún medio digital quiere vivir al mar-} gen de las redes sociales

La relación de Twitter con los acontecimientos actuales ha incentivado el estudio de esta red social. Los trabajos se han ocupado tanto de la metodología para su estudio (Bruns; Burgess, 2011) como sobre el papel de Twitter en la difusión de noticias (Hermida, 2010; Stassen, 2010; Bruns, 2011; Holton; Lewis, 2011; Vis, 2013; Hermida, 2013; Larson; HaIlvard, 2015; Arrabal-Sánchez; Aguilera-Moyano, 2016).

Por su parte, la otra red más estudiada, Facebook, aunque su uso no está tan generalizado en la industria mediática y en el ámbito periodístico, se ha analizado en los últimos años desde las características de sus usuarios (Sun; Rosenn; Marlow; Lento, 2009) hasta por qué la utilizan o el efecto de red global, con las posibilidades que ofrece y el poder que tiene (Kirkpatrick, 2010) o la presencia de medios de diferentes países europeos e iberoamericanos y su uso periodístico (Noguera-Vivo, 2010; García-de-Torres et al., 2011; Bakshy; Rosenn; Marlow; Adamic, 2012; González-Molina; Ramos-del-Cano, 2013; Mitchell; Page, 2013).

A estos estudios acerca de las redes sociales y los medios de comunicación hay que añadir la revisión de artículos científicos publicados en la última década que analizan la difusión de noticias y los medios sociales (Kümpel; Karnowski; Keyling, 2015). Dicho análisis muestra una aproximación desde muy diferentes ámbitos científicos, con trabajos de investigadores de diferentes procedencias que han focalizado los estudios empíricos.

La mayoría de esas investigaciones coincide no sólo en el masivo empleo de las redes sociales para la difusión de las 
Tabla 1. Twitter y YouTube son las redes de mayor impacto en los medios

\begin{tabular}{|l|c|c|c|}
\hline \multicolumn{1}{|c|}{ Respuesta } & Nada (\%) & Poco (\%) & Mucho (\%) \\
\hline Facebook & 10,4 & 26,0 & 63,5 \\
\hline Twitter & 3,0 & 9,9 & $\mathbf{8 7 , \mathbf { 1 }}$ \\
\hline LinkedIn & 13,4 & 35,1 & 51,1 \\
\hline Instagram & 6,3 & 32,3 & 61,5 \\
\hline YouTube & 3,0 & 13,9 & $\mathbf{8 3 , 2}$ \\
\hline Pinterest & 20,9 & 31,9 & 47,3 \\
\hline Otras & 15,4 & 23,1 & 61,5 \\
\hline
\end{tabular}

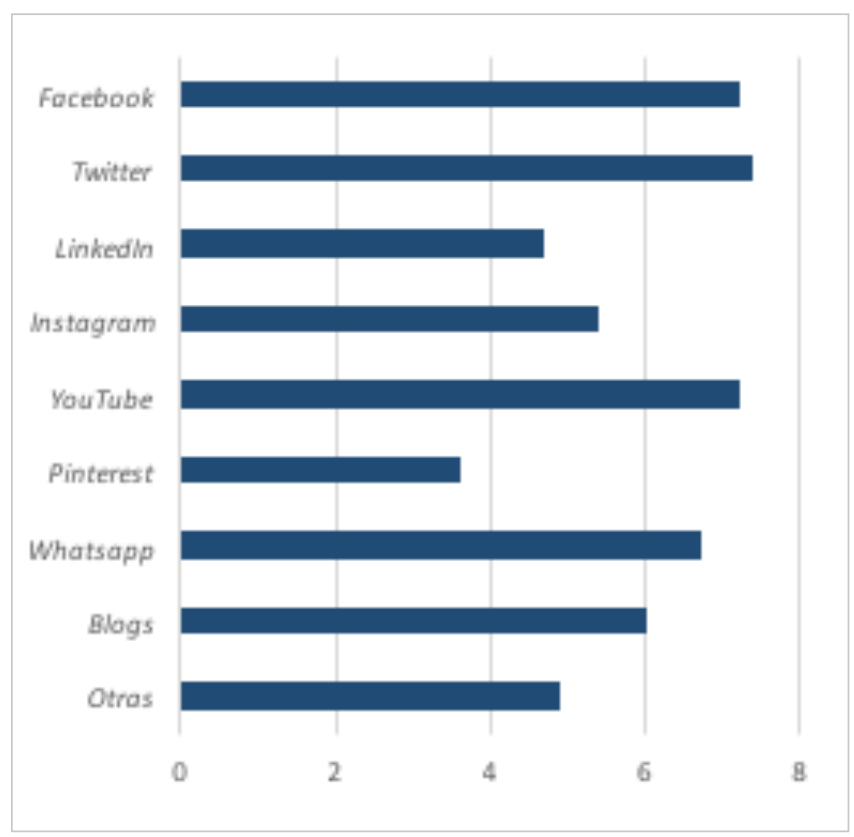

Elaborado a partir del Barómetro de gestión de medios de comunicación, Xescom, 2015.

noticias sino que, gracias a las estrategias de gestión, se aprecia una interrelación o efecto polinización (cross-pollination) en la difusión de contenidos a través de las diferentes redes. Los datos, aunque muestran que muchos aspectos todavía deben investigarse más, indican, como criterio general coincidente, que los profesionales de la industria de la comunicación deben conocer y manejarse con soltura en el empleo de las redes sociales.

\section{Redes en las empresas de comunicación}

La gestión de las redes sociales por parte de las empresas de comunicación en España es el objeto de nuestro estudio, situado en un contexto de la sociedad en red (Castells, 1996), donde el papel de los usuarios en la generación de contenidos y su comercialización resulta central en los nuevos usos y consumos (Ritzer; Jurgenson, 2010). Las organizaciones, que se interesan por la evolución del papel de las noticias en Twitter y Facebook (Barthel; Sheaver; Gottfried; Mitchell, 2015) se apoyan cada vez más en la comunicación móvil y en las redes sociales para alcanzar a la audiencia (Mitchell; Page, 2015).

Los esfuerzos se centran, a pesar de los riesgos, en sacar más provecho a las redes sociales para construir y mante- ner las audiencias (Lee, 2015). La gestión que hacen las organizaciones mediáticas en España de las redes sociales la hemos analizado en el momento en el que los profesionales nativos digitales están en disposición de acceder a su trabajo en la industria de la comunicación. El objetivo es disponer de datos sobre uso, valoración y posibilidades de las redes sociales para el futuro del periodismo.

\section{El valor que la propia red Twitter no con- sigue captar para sí, lo retienen los me- dios que viralizan sus contenidos a tra- vés de ella}

\section{Resultados del Barómetro de medios}

Como se puede ver en la tabla 1, los gestores de los medios de comunicación españoles consideran que Twitter, YouTube y Facebook son las redes que han impactado más en sus actividades y negocios. Esa es la respuesta dominante en la primera pregunta en la que se planteaba si las redes sociales digitales habían cambiado los medios de comunicación tradicionales. En la categoría "Otras" aparecen respuestas relacionadas con redes profesionales, de mensajería y telefonía social (Whatsapp, Skype, Viber, etc.). El cruce de las respuestas por medios y sectores indica que lo que priorizan las empresas es la recuperación de valor que pueden obtener en cada red.

La siguiente pregunta fue: ¿Cuál de las redes cree que le aporta o puede aportar en el futuro más valor a su medio y/o organización de comunicación? Valore de 1 a 10 (teniendo en cuenta que 1 es poco y 10 mucho). En este caso, como refleja la tabla 2, sobresale de nuevo Twitter.

Las teorías del valor y del capital social son esenciales para la investigación de los medios, metamedios y redes. Las empresas así lo perciben y no dudan, a la hora de valorar las redes, a aquellas que consideran de más utilidad para conservar y proyectar su valor más allá de sus modelos de negocio tradicionales. En primer lugar sitúan a Twitter y esa opinión es coherente con respecto a la apropiación de valor entre ellos y la propia red.

Tabla 2. Twitter destaca en la aportación de valor por los medios

\begin{tabular}{|l|c|}
\hline \multicolumn{1}{|c|}{ Respuestas } & Puntuación media \\
\hline Facebook & 7,2 \\
\hline Twitter & 7,4 \\
\hline Linkedln & 4,7 \\
\hline Instagram & 5,4 \\
\hline YouTube & 7,2 \\
\hline Pinterest & 3,6 \\
\hline Whatsapp & 6,7 \\
\hline Blogs & 6,0 \\
\hline Otras & 4,9 \\
\hline
\end{tabular}

Elaborado a partir del Barómetro de gestión de medios de comunicación, Xescom, 2015. 
Tabla 3. Las redes y el futuro del periodismo

\begin{tabular}{|l|c|c|c|}
\hline \multicolumn{1}{|c|}{ Respuesta } & Nada (\%) & Poco (\%) & Mucho (\%) \\
\hline Facebook & 5,9 & 18,8 & 75,2 \\
\hline Twitter & 4,0 & 15,8 & $\mathbf{8 0 , 2}$ \\
\hline LinkedIn & 12,6 & 41,1 & 46,3 \\
\hline Instagram & 7,7 & 35,2 & 57,1 \\
\hline YouTube & 5,2 & 15,5 & 79,4 \\
\hline Pinterest & 19,0 & 34,5 & 46,4 \\
\hline Otras & 16,0 & 25,3 & 58,7 \\
\hline
\end{tabular}

Elaborado a partir del Barómetro de gestión de medios de comunicación, Xescom, 2015.

Curiosamente, es Twitter la red social global que más problemas de modelo propio de negocio tiene porque, aunque crea y proyecta valor, no es capaz de captarlo para sí misma, como lo hacen Facebook o YouTube, que incrementan al mismo ritmo ingresos de publicidad y registro de interacciones de los big data en sus plataformas digitales. La conclusión es que el valor que no capta Twitter, lo retienen los medios que viralizan sus contenidos a través de ella.

Los profesionales de la industria de la comunicación deben conocer y manejarse con soltura en el empleo de las redes sociales

La tercera pregunta de la encuesta que nos interesa para este artículo es la relación de las redes con el futuro del periodismo. La pregunta fue planteada de la siguiente forma: ¿Cree que las redes sociales están cambiando o cambiarán en el futuro inmediato el periodismo?

De nuevo volvemos a hallar la priorización de Twitter y YouTube en las respuestas de los responsables de las empresas de comunicación. A este respecto hay que matizar que, a pesar de que la encuesta fue dirigida a máximos responsables de gestión de las empresas de comunicación, una parte importante (más del 40\%) de los que respondieron fueron personas relacionadas con la dirección periodística de los medios. En el apartado "Otras", volvemos a encontrarnos con las redes especializadas, profesionales, de mensajería social, de verificación de datos y del ámbito periodístico.

\section{Conclusiones}

Los responsables de las empresas de comunicación tradicionales coinciden a la hora de valorar las redes sociales como instrumentos que conservan y proyectan su valor más allá de sus negocios tradicionales. De los resultados de la encuesta y del análisis de las respuestas se concluye que existe una opinión mayoritaria entre los gestores de los medios de comunicación españoles que considera que las redes Twitter, YouTube y Facebook son las que han impactado más positivamente en sus actividades y negocios.

Como ya apuntamos anteriormente, a partir del análisis de los expertos en medios digitales se concluye que el valor y el capital social, con todos sus respectivos atributos (popularidad, reputación, prestigio, confianza, credibilidad), hay que situarlos en el eje prioritario de la gestión y la investigación de los medios y los metamedios. Son líneas de investigación clásicas y veteranas de la sociología, psicología, antropología y otras ciencias aplicadas al estudio de las redes sociales presenciales, pero que aún no se han prodigado sobre estas nuevas herramientas de las ciencias de la comunicación. A esas líneas podemos agregar también los desafíos del estudio de la participación e interacción (Carpentier, 2016), los nuevos lenguajes y formatos transmedia, las relaciones con la economía política, las cuestiones éticas y deontológicas.

En la actual eclosión de investigación sobre las redes digitales y los medios de comunicación ya empiezan a entrar varias de esas cuestiones; y es deseable que se prodiguen más. La incorporación de las nuevas metodologías de metadatos al estudio de la meta comunicación y los metamedios ayudará, sin duda, a avanzar más en esas líneas y a obtener resultados de utilidad para la aplicación a la transferencia de la innovación en los contenidos, los modelos de negocio y las políticas de comunicación.

Señalar, por último, las limitaciones con respecto a nuestro Barómetro de gestión de medios y a este artículo. Esta investigación es todavía un work in progress, porque es necesario profundizar más en el campo y la encuesta requiere más ediciones y series para su categorización así como complementación con otros métodos cualitativos. Son futuras líneas abiertas por la Red Xescom y por quien desee conectar con ellas.

Los gestores de los medios de comunicación españoles consideran que Twitter, YouTube y Facebook son las redes que han impactado más en sus actividades y negocios

\section{Mención de reconocimiento}

Los resultados de este artículo forman parte de las actividades de investigación promovidas a través de la Red Xescom (R2014/026 Xescom), apoyada por la Consellería de Cultura, Educación e Ordenación Universitaria de la Xunta de Galicia; de las tareas exploratorias de los proyectos del Programa Estatal de Fomento de la Investigación Científica y Técnica de Excelencia, subprograma estatal de Generación de Conocimiento del Ministerio de Economía y Competitividad de España sobre "Indicadores de gobernanza, financiación, rendición de cuentas, innovación, calidad y servicio público de las RTV europeas aplicables a España en el contexto digital" (referencia CSO2015-66543-P) y "Usos y preferencias informativas en el nuevo mapa de medios en España: modelos de periodismo para dispositivos móviles" (referencia: CSO2015-64662-C4-4-R); así como del Programa Prometeo de la Secretaría Nacional de Educación Superior, Ciencia, Tecnología e Innovación (Senescyt) de Ecuador, desarrollado en las Universidades Técnica Particular de Loja (UTPL) y Pontificia Universidad Católica de Ibarra (Pucesi). 


\section{Bibliografía}

Allee, Verna (2009). "Value creating networks: Organizational issues and challenges". The learning organization, v. 6, n. 6, pp. 427-442.

http://citeseerx.ist.psu.edu/viewdoc/download?doi=10.1.1. 469.384\& rep $=$ rep1\&type $=p d f$

http://dx.doi.org/10.1108/09696470910993918

Arrabal-Sánchez, Gabriel; De-Aguilera-Moyano, Miguel (2016). "Comunicar en 140 caracteres. Cómo usan Twitter los comunicadores en España”. Comunicar, v. 24, 46, pp. 9-17. http://dx.doi.org/10.3916/C46-2016-01

Bakshy, Eytan; Rosenn, Itamar; Marlow, Cameron; Adamic, Lada (2012). "The role of social networks in information diffusion". En: Proceedings of the ACM Conference on the World Wide Web, Lyon, France.

http://cameronmarlow.com/media/bakshy-the_role-2012b.pdf

Barthel, Michael; Sheaver, Elisa; Gottfried, Jeffrey; Mitchell, Amy (2015). "The evolving role of news on Twitter and Facebook". Pew Research Center, Journalism \& media, July 14. http://www.journalism.org/2015/07/14/the-evolving-roleof-news-on-twitter-and-facebook

Bateson, Gregory (1973). Steps to an ecology of mind: Collected essays in anthropology, psychiatry, evolution and epistemology. Londres: Granada. ISBN: 0876689500

Beer, David (2008). "Social network(ing) sites... revisiting the story so far: A response to Danah Boyd \& Nicole Ellison". Journal of computer-mediated communication, v. 13, pp. 516-529. http://dx.doi.org/10.1111/j.1083-6101.2008.00408.x

Boyd, Danah M.; Ellison, Nicole B. (2007). "Social network sites: Definition, history, and scholarship". Journal of computer-mediated communication, v. 13, n. 1, pp. 210-230. http://dx.doi.org/10.1111/j.1083-6101.2007.00393.x

Bruns, Axel (2011). "Twitter events in perspective (updated). Mapping online publics".

http://mappingonlinepublics.net/2011/02/10/twitterevents-in-perspective

Bruns, Axel; Burgess, Jean (2011). "Researching news discussion on Twitter: New methodologies". The future of journalism, 8-9.

http://cs.wellesley.edu/ trails/retweetpapers/papers/ NewsDiscussion.pdf

Campos-Freire, Francisco (2015a). "Adaptación de los medios tradicionales a la innovación de los metamedios". El profesional de la información, v. 24, n. 4, p. 441-450. http://dx.doi.org/10.3145/epi.2015.jul.11

Campos-Freire, Francisco (2015). "Los sitios de redes sociales como paradigma del ecosistema digital”. En: CamposFreire, Francisco; Rúas-Araujo, José (eds.). Las redes sociales digitales en el ecosistema mediático. Cuadernos artesanos de comunicación. Sociedad Latina de Comunicación Social, pp. 13-36. ISBN: 9788416458233

http://www.cuadernosartesanos.org/abscac92.html http://dx.doi.org/10.4185/cac92

Carpentier, Nico (2016). "Beyond the ladder of participation: An analytical toolkit for the critical analysis of participatory media processes". Javnost-The public, v. 23, n. 1, pp. 70-88. http://dx.doi.org/10.1080/13183222.2016.1149760

Carrington, Peter J.; Scott, John; Wasserman, Stanley (2005). Models and methods in social network analysis. Cambridge University Press. ISBN: 9780521600972

Castells, Manuel (1996). The information age: Economy, society and culture. Vol. I: The rise of the network society. Oxford: Blackwell. ISBN: 9781405196864

Christakis, Nicholas A.; Fowler, James H. (2009). Connected: The surprising power of our social networks and how they shape our lives. New York: Little, Brown and Co. http://connectedthebook.com/pdf/excerpt.pdf

Cision (2015). 2015 Global social journalism study. http://www.cision.com/us/resources/white-papers/2015global-social-journalism-study

Coleman, James S. (1988). "Social capital in the creation of human capital". The American journal of sociology, v. 94, pp. 1531-1547.

https://www.jstor.org/stable/2780243

Dollé, Nathalie (2013). "Journalistes et réseaux sociaux. Evolution ou révolution?".

http://www.alliance-journalistes.net/IMG/pdf/_journalistes et_reseaux_sociaux_int_site.pdf

Donders, Karen; Raats, Tim (2012). "Measuring public value with the public value test: Best of worst practice?". En: K. Janssen \& J. Comprovoets (eds.), Geographic data and the law. Defining new changes. Leuven University Press. ISBN: 9788415698982

Ellison, Nicole B.; Vitak, Jessica; Gray, Rebecca; Lampe, Cliff (2014). "Cultivating social resources on social network sites: Facebook relationship maintenance behaviors and their role in social capital processes". Journal of computer-mediated communication, v. 19 , n. 4, pp. 855-870.

http://dx.doi.org/10.1111/jcc4.12078

Farhi, Paul (2009). "The Twitter explosion". American journalism review.

http://ajrarchive.org/Article. asp? $i \mathrm{id}=4756$

Gangadharbatla, Harsha (2008). "Facebook me: Collective selfesteem, need to belong, and internet self-efficacy as predictors of the I generation's attitudes toward social networking sites". Journal of interactive advertising, v. 18, n. 2, pp. 5-15. http://dx.doi.org/10.1080/15252019.2008.10722138

García-Canclini, Néstor (1989). Culturas híbridas: Estrategias para entrar y salir de la modernidad. México: Grijalbo. ISBN: 9684199546

García-de-Torres, Elvira; Rost, Alejandro; Edo, Concha; Said, Elías; Arcila, Carlos; Sánchez, Jorge; Yezers'ka, Lyudmyla; Calderín, Mabel; Rojano, Miladys; Jerónimo, Pedro; Serrano, Ana; Corredoira, Loreto (2011). "Uso de Twitter y Facebook por los medios iberoamericanos. El profesional de la información, v. 20, n. 6, pp. 611-620.

http://recyt.fecyt.es/index.php/EPI/article/view/epi.2011.nov.02

García-Estévez, Noelia (2013). “Presencia de las redes sociales y medios de comunicación: representación y partici- 
pación periodística en el nuevo contexto social". Universidad de Sevilla. Tesis doctoral.

http://fondosdigitales.us.es/tesis/tesis/2336/presencia-de-lasredes-sociales-y-medios-de-comunicacion-representacion-yparticipacion-periodistica-en-el-nuevos-contexto-social

González-Molina, Sonia; Ramos-del-Cano, Fátima (2013). "El uso periodístico de Facebook y Twitter: un análisis comparativo de la experiencia europea. Historia y comunicación social, v. 8, pp. 419-433.

http://revistas.ucm.es/index.php/HICS/article/view/44253/41816

Gordon, Raymond (2003). "The meanings and implications of convergence". En Kawamoto, K. (ed.), Digital journalism: Emerging media and the changing horizons of journalism. EUA: Rowman y Littlefield Publishers. ISBN: 10: 0742526801

Greenhow, Christine; Reifman, Jeff (2009). "Engaging youth in social media. Is Facebook the new media frontier?". Nieman report. Nieman Foundation for Journalism at Havard. http://niemanreports.org/articles/engaging-youth-insocial-media-is-facebook-the-new-media-frontier

Guitton, Matthieu J. (2012). "The immersive impact of meta-media in a virtual world". En Computers in human behavior, v. 28, n. 2, pp. 450-455.

http://dx.doi.org/10.1016/j.chb.2011.10.016

Hargittai, Eszter (2007). "Whose space? Differences among users and non-users of social network sites". Journal of computer-mediated communication, v. 13, n. 1, pp. 1-19. http://www.it.uu.se/edu/course/homepage/avint/vt09/6.pdf

Harper, Ruth A. (2010). "The social media revolution: Exploring the impact on journalism and news media organizations". Studentpulse.com.

http://www.studentpulse.com/articles/202/the-socialmedia-revolution-exploring-the-impact-on-journalism-andnews-media-organizations

Hermida, Alfred (2010). "Twitering the news". Journalism practice, v. 4, n. 3, pp. 297-308.

http://dx.doi.org/10.1080/17512781003640703

Hermida, Alfred (2013). "\#Journalism: Reconfiguring journalism research about Twitter, one tweet at a time". Digital journalism, v. 1, n. 3, pp. 295-313.

http://dx.doi.org/10.1080/21670811.2013.808456

Herrero, Eva (2013). "Periodistas y redes sociales en España: del 11M al 15M (2004-2011)". Madrid: Universidad Carlos III. Tesis doctoral.

http://e-archivo.uc3m.es/handle/10016/17648\#preview

Holton, Avery E.; Lewis, Seth C. (2011). "Journalists, social media and the use of humor on Twitter". Cios/The electronic journal of communications.

http://www.cios.org/EJCPUBLIC/021/1/021121.html

International Telecommunication Union -ITU- (2014). "Mesuring the information society report 2014". http://www.itu.int/dms_pub/itu-d/opb/ind/D-IND-ICTOI2014-SUM-PDF-E.pdf

Jenkins, Henry (2006). Convergence culture: Where old and new media collide. NY: New York University Press. ISBN: 0 814742815
Jensen, Klaus-Bruhn (2010). Media convergence: The three degrees of network, mass, and interpersonal communication. Londres: Routledge. ISBN: 9780415482042

Jensen, Klaus-Bruhn (2013). "How to do things with data: Meta-data, meta-media, and meta-communication". First Monday, v. 18, n. 10.

http://firstmonday.org/article/view/4870/3751

Kay, Alan; Goldberg, Adele (1977). "Personal dynamic media". Journal computer, v. 10, n. 3, pp. 31-41.

http://www.vpri.org/pdf/m1977001_dynamedia.pdf

Kim, Daejoong; Kim, Jang-Hyun; Nam, Yoonjae (2014). "How does industry use social networking sities? An analysis of corporate dialogic uses of Facebook, Twitter, YouTube, and Linkedln by industry type". Quality \& quantity, v. 48, n. 5, pp. 2605-2614.

http://dx.doi.org/10.1007/s11135-013-9910-9

Kirkpatrick, David (2010). The Facebook effect: The inside story of the company that is connecting the world. New York: Virgin Books. ISBN: 0753522748

Kümpel, Anna-Sophie; Karnowski, Veronika; Keyling, Till (2015). "News sharing in social media: A review of current research on news sharing users, content, and networks. $S M+S$ Social media+society, v. 2, n. 1, pp. 1-14.

http://sms.sagepub.com/content/1/2/2056305115610141. full.pdf+html

Larson, Anders-Olof; Hallvard, Moe (2015). "Bots or journalists? News sharing on Twitter". Communications, v. 40, n. 3, pp. 361-370.

http://www.andersoloflarsson.se/wp-content/uploads/2015/09/ commun-2015-0014.pdf

Lasorsa, Dominic L.; Lewis, Seth C.; Holton, Avery E. (2011). "Normalizing Twitter: Journalism practice in an emerging communication space." Journalism studies, v. 13, n. 1, pp. 19-36. http://dx.doi.org/10.1080/1461670X.2011.571825

Latour, Bruno (1987). Science in action: How to follow scientists and engineers through society. Cambridge: Harvard University Press. ISBN: 0674792912

Latour, Bruno (1993). We have never been modern. Harvard Univ. Press. ISBN: 0674948386

http://monoskop.org/images/e/e4/Latour_Bruno_We_ Have_Never_Been_Modern.pdf

Lee, Chei Sian; Ma, Long (2012). "News sharing in social media: The effect of gratifications and prior experience". Computers in human behavior, v. 28, n. 2, pp. 331-339. http://dl.acm.org/citation.cfm ?id=2109078

Lee, Jayeon (2015). "The double-edged sword: The effects of journalists' social media activities on audience perceptions of journalists and their news products. Journal of computer-mediated communication, v. 20, n. 3, pp. 312-329. http://dx.doi.org/10.1111/jcc4.12113/full

Lin, Nan (2001). Social capital: A theory of social structure and action. London: Cambridge University Press. ISBN: 052152167X

Lowery, Countney (2009). “An explosion prompts rethinking 
of Twitter and Facebook". Nieman Reports. Nieman Foundation for Journalism.

http://niemanreports.org/articles/an-explosion-promptsrethinking-of-twitter-and-facebook

http://dx.doi.org/10.1111/jcc4.12113/full

Manovich, Lev (2001). The language of new media. Cambridge, MA: MIT Press. ISBN: 9780262133746

Manovich, Lev (2005). El lenguaje de los nuevos medios de comunicación: la imagen en la era digital. Barcelona: Paidós. ISBN: 9788449317699

https://uea1arteycomunicacion.files.wordpress.com/2013/09/ manovich-el-legunaje-de-los-nuevos-medios.pdf

Manovich, Lev (2008). Software takes command. New York: Georgetown University.

http://faculty.georgetown.edu/irvinem/theory/ManovichSoftware-Takes-Command-ebook-2008-excerpt.pdf

Manovich, Lev (2015). “Understanding meta-media”. Ctheory. http://journals.uvic.ca/index.php/ctheory/article/ view/14459/5301

McLuhan, Marshall (1964). Understanding media: The extensions of man. New York: New American Library. ISBN: 0262631598

Mitchell, Amy; Page, Dana (2015). State of the news media 2015. http://www.journalism.org/2015/04/29/state-of-the-newsmedia-2015

Nadkami, Ashwini; Hofmann, Stefan G. (2012). "Why do people use Facebook? Personality and individual differences". National Center for Biotechnology Information.

http://www.ncbi.nlm.nih.gov/pmc/articles/PMC3335399

Noguera-Vivo, José-Manuel (2010). “Redes sociales como paradigma periodístico. Medios españoles en Facebook". Revista latina de comunicación social, n. 65, pp. 176-186.

http://www.revistalatinacs.org/10/art/891_UCAM/13_JM_ Noguera.html

O'Reilly, Tim (2004). "What is web 2.0".

http://www.oreilly.com/pub/a/web2/archive/what-isweb-20.html

Oriella PR Network (2011). "The state of journalism in 2011-Oriella PR Network digital journalism study".

http://orielladigitaljournalism.com/view-report.htm/

Paulussen, Steve; Harder, Raymond A. (2014). "Social media references in newspapers. Facebook, Twitter and YouTube as sources in newspapers journalism". Journalism practice, v. 8, n. 5, pp. 542-551.

http://dx.doi.org/10.1080/17512786.2014.894327

Pew Research Center (2015). "Social media usage: 2005-2015". http://www.pewinternet.org/2015/10/08/socialnetworking-usage-2005-2015

Prensky, Marc (2001). "Digital natives, digital immigrants". On the horizon, v. 9, n. 5, pp. 1-6.
http://www.marcprensky.com/writing/Prensky\%20-\%20 Digital\%20Natives,\%20Digital\%20Immigrants\%20-\%20Part1. $p d f$

Reuters Institute (2015). Digital news report 2015. http://www.digitalnewsreport.org

Ritzer, George; Jurgenson, Nathan (2010). “Production, consumption, prosumption. The nature of capitalism in the age of the digital 'prosumer'". Journal of consumer culture, v. 10, n. 1 , pp. 13-36.

http://joc.sagepub.com/content/10/1/13.abstract

Stassen, Wilma (2010). "Your news in 140 characters: Exploring the role of social media in journalism". Global media journal, African edition, v. 4, n. 1, pp. 1-16.

http://dx.doi.org/10.5789/4-1-15

Stenger, Thomas (2009). "Social network sites (SNS): do they match? Definitions and methods for social sciences and marketing research". En XXIX Conf. Insna, San Diego, EUA. http://www.academia.edu/2521387/Social_Network_ Sites_SNS_do_they_match_Definitions_and_methods_for_ social_sciences_and_marketing_research

Suárez-Candel, Roberto (2012). “Adapting public service to the multiplatform scenario: Challenges, opportunities and risks". Working papers of the Hans Bredow Institute, 25. Hamburgo: Hans Bredow Institute for Media Research. ISBN: 9783872961198

https://www.hans-bredow-institut.de/webfm_send/661

Sun, Eric; Rosenn, Itamar; Marlow, Cameron A.; Lento, Thomas M. (2009). "Gesundheit! Modeling contagion through Facebook news feed. Proceedings of the $3^{\text {rd }}$ Int/ ICwsm conf, pp. 146-153, San Jose, CA.

http://cameronmarlow.com/media/sun-2009-modelingsocial-contagion.pdf

Tambini, Damian (2015). "Public media and digitisation: Seven theses". En: Arriaza Ibarra, Karen; Novak, Eva; Kuhn, Raymond: Digital journalism: Making news, breaking news. ISBN: 978138017856

Thelwall, Mike (2008). "No place for news in social network web sites?". Online information review, v. 32, n. 6, pp. 726744.

http://dx.doi.org/10.1108/14684520810923908

Veloso, Ana-Sofia (2015. "O uso das redes sociais por jornalistas em Portugal". Sur le journalisme, About journalism, Sobre jornalismo, v. 4, n. 1, pp. 120-139.

http://surlejournalisme.com/rev/index.php/slj/article/ view/204/90

Vis, Farida (2013). "Twitter as a reporting tool breaking news". Digital journalism, v. 1, n. 1, pp. 27-47. http://dx.doi.org/10.1080/21670811.2012.741316

Willnat, Lars; Heaver, David H. (2014). "The American journalist in the digital age: Key findings".

http://news.indiana.edu/releases/iu/2014/05/2013american-journalist-key-findings.pdf 